ISSN 1112-9867

http://www.jfas.info

\title{
WHITE LIGHT SIGNAL SIMULATOR MICROCONTROLLER DESIGN
}

\author{
B. Haghighi ${ }^{1}$, N. Ebrahimi ${ }^{1}$ and N. Haghighi ${ }^{2, *}$ \\ ${ }^{1}$ Dept. of Physics, Faculty of Science, Islamic Azad University, Mashhad Branch, Mashhad, \\ Iran \\ ${ }^{2}$ Faculty Of Science, Islamic Azad University, Qum Branch, Qum, Iran
}

Published online: XX June 2016

\begin{abstract}
In this research, first through experimental studies, the recorded signals associated with the light white from human retina cells were digitized, then the digital data were calculated and the resulted mathematical equation was programmed on a microcontroller, and by designing a circuit, the output voltage over time similar to initial signals associated to the white light was obtained.This circuit can be designed and implemented on an IC, which can be planted on retina cells, so that the brain can receive signals analogous to the signals associated with the white light.
\end{abstract}

Keywords: retina; nervous signals; optical receivers.

Author Correspondence, e-mail: haghighi_236@mail.ru

doi: http://dx.doi.org/10.4314/jfas.8vi2s.59

\section{INTRODUCTION}

The eyes are the organs of vision with triple layers and relatively spherical structure that is specialized to receive light and give respond to it. Light enters the eye through the cornea, breaks through aqueous humor, and reaches the lens. The lens adjustment (adaptation) focuses the light on the retina by passing it through vitreous. In retina, the light-sensitive cells (cone cells, rod cells) send nerve impulses through the optic nerve to the brain. The light rays from the 
object convert to the electrical message after hitting the retina. The message is transmitted by the optic nerve. The two optic nerves meet in the central part of the brain, called the optic chiasm. Half of each of two optic fibers intersect with each other and then continue on their way. Neural messages reach the cortex in the brain hemispheres. The cells of the back of the brain decode these messages and create the image. This way you'll be able to see [1].

\section{ELECTRIC SIGNALS ORIGINATINGFROM THE EYES}

Eyes are the center of a fixed electric potential field, the field that is completely irrelevant to light stimulation. In fact, it is possible to identify this field in full darkness / or with closed eyes. It can be described as a fixed bipolar with the positive pole at cornea and the negative pole at retina. The potential difference and eye rotation are the basis of a signal to be measured on a pair of electrodes on the surface of the eyes. The electroretinogram (ERG) is clinically recorded using a special contact lens that is placed on the cornea is recorded, and contains a chloride silver wire. Electrode is placed in the cornea [2].
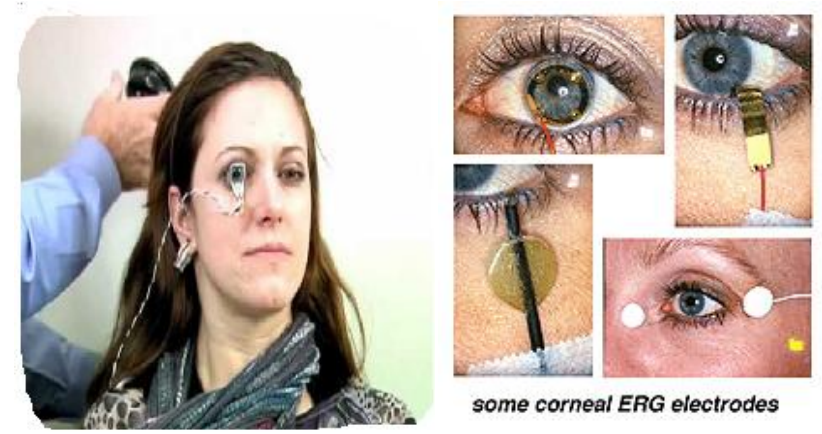

Fig.1. The ERG test for overall retina response to light stimulus by a potential difference between the two electrodes

Contact lens electrode or active electrode is placed on the cornea after anesthesia. The electrode is directly responsible to stimulate the retina. Ground electrode is attached on the patient's ear with a special paste. The role of this electrode is to remove noise during the test. Reference electrode or inactive electrode is attached on the forehead, slightly above the upper margin of the orbit. The electrode can also be attached on the mastoid bone. This electrode acts as a negative pole, so it is placed close to the posterior pole of the eye node (indifferent electrode) $[5]$ 
The reference electrode is usually placed on the forehead, temporal, or earlobe. ERG source is created in different layers of the retina. Therefore, these sources are distributed and are placed in a conductive volume that contains the eye, eyeball, and to some extent, all of the head. Record electrodes are in this area. For ERG, you can identify gradual layer changes, of which different parts of the wave are formed, that begin by a brief light flash stimulus of the receptor. Graph below shows retina cells and their response to a white light flash point. In figure 2, the chart displays rod and cone receptors, of which the receptor potential is obtained negative. This cell converts the bipolar to either depolarized or hyperpolarized. Amacrine cells have a negative feedback effect. Ganglion cells burn an action pulse so that the rod result is proportional with the stimulus light level, and internal layer of retina cells have Muller cells. Passing potential from Müller cells depend on its Nernst potassium potential, which is influenced by changes in potassium levels outside the cells. Müller cells can help the b-wave of both cone and rod receptor [3].

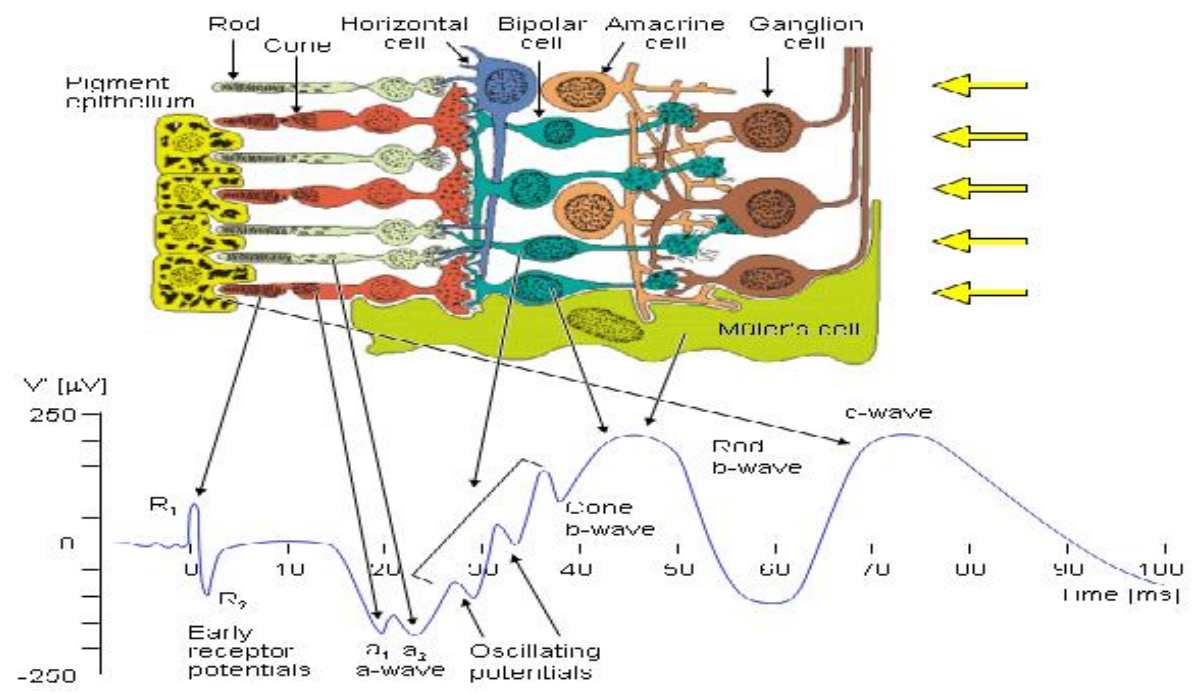

Fig.2. Signal is produced by initial changes in photo pigment molecule of the receptor considering light action

1. The first signal is produced by initial changes in photo pigment molecule of the receptor considering action of light. This usually cause R1 positive deviation following an R2 negative deviation, together which produce initial receptor potential.

2. Negative wave "a", which stems from the photoreceptors of the eye. 
3. Changes in waves "a" and "b" in adaptation to darkness conditions in response to minimum light intensity and increasing amplitude both a and b waves as well as ' $a$ ' wave becoming two-phase, and oscillatory potentials in the ascending curve of "b" wave in response to the increasing intensity of stimulation, can be seen.

4. Positive wave "b" which is made by the layer of bipolar cells and strongly from muller cells.

5. Positive wave "c" that originates in the pigmented epithelium layer cells, although stimulating the pupil by the light at the time of test could also produce this wave. [4]

Cells of retina and their response to a point light flash. Receptor is rod and cone in which a negative receptor potential is obtained. This cell converts the bipolar to either depolarized or hyperpolarized. Amacrine cells have a negative feedback effect. Ganglion cells burn an action pulse so that the result is a rod chain proportional with the stimulus light level. The second maximum, which is positive, is a "b" wave. To explain its source, it should be noted that Muller cells exist in internal layers of retina. These are glial cells and have no synaptic relationship with retina cells. Passing potential from Müller cells depends on its Nernst potassium potential, which is influenced by changes in potassium levels outside the cells. In the second case, when the receptors are stimulated, the propagation increases with potassium. In addition, action pulse of lymph cells takes place as the potassium flows (the result of these events creates Muller cell response, the latter is the source of "b" wave. Müller cells can help b wave both from cone and rod receptors separately. C - wave is positive like b - wave, positive wave "c" originating from pigmented epithelium layer cells, although stimulating the pupil by light at the time of the test could also produce this wave. The shown oscillatory potentials are small pulse waves that appear in b - wave adapted with light [2].

\section{ANALYSIS OF THE PROBLEM}

Using the recorded sample and selecting the signals associated with the white light and using Getdata software, we extracted signal data and obtain the equation of each curve:

The first curve ranges between 1-10. The first function is: 
(1) $\quad \mathrm{Y} \_1=(-2.4398) * \sin ((2.11912) * \mathrm{x})-2.23613$

The associated graph is defined as follows:

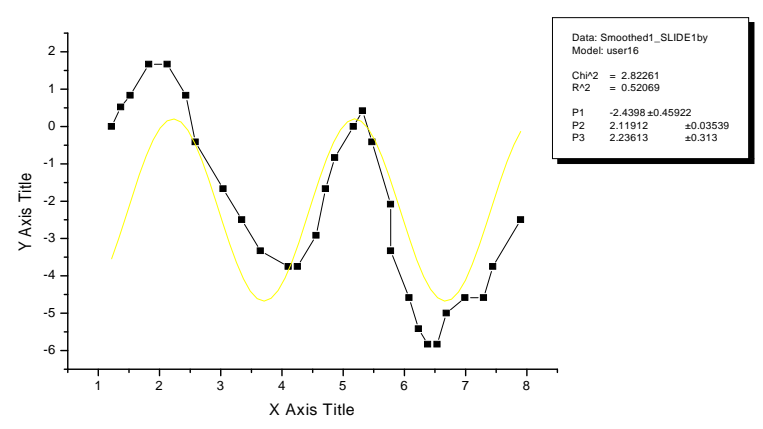

Fig.3. Section one

The second curve ranges between 10-20, and the graph of section 2 with the function

$$
\text { (2) } \quad Y \_2=11.31258+(-121.60425) /(0.78231 * \sqrt{ }(\pi / 2)) e^{\wedge}(-2
$$$$
\llbracket\left((x-10.08356) \rrbracket^{\wedge} 2 /\left(\left([0.78231) \rrbracket^{\wedge} 2\right)\right)+0.00035 * e^{\wedge}(x / 0.71944)\right.
$$

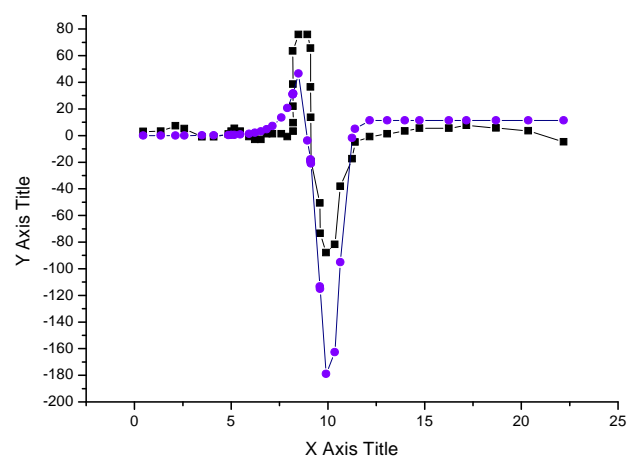

Fig.4. Second part

The third curve ranges between 20-50, for the third function with the following equation:

(3) $\quad y \_3=2185.43432-160.98492 * X+2.75861 * X 2$

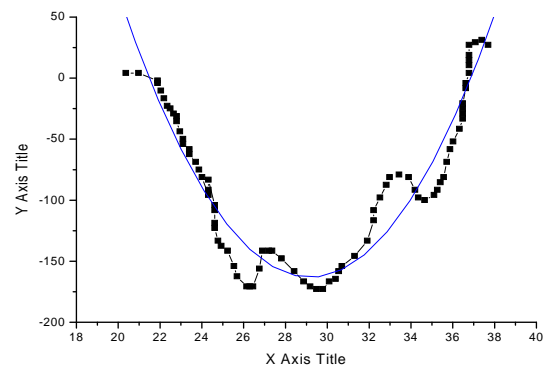

Fig.5. Section 3 
The fourth curved ranges between 50-100, and the fourth section of its graph is a function with the following equation:

$$
\text { (4) } \quad y_{-} 4=-138.01066+\cos (0.1986 * x)+74.7230
$$

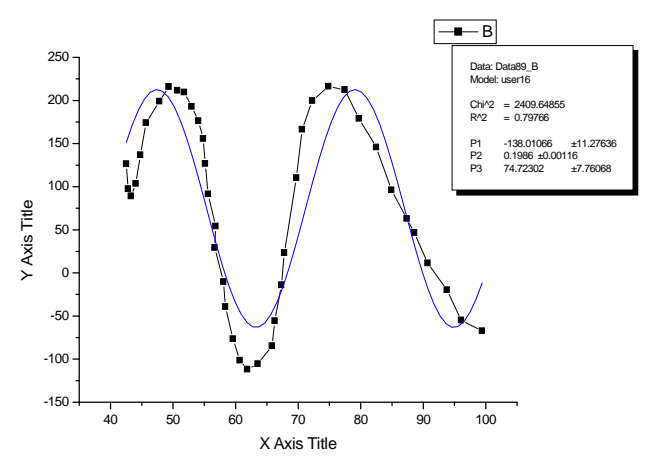

Fig.6. Section four

By doing the above calculations, signal diagram function can be obtained as the following:

(5) $\quad Y_{\text {tot }}=-138.01066+\cos (0.1986 * x)+74.72302+2185.43432-160.98492 *$

$X+2.75861 * X^{2}+11.31258+\frac{-121.60425}{0.78231 * \sqrt{\frac{\pi}{2}}}+e^{-2 \frac{(x-10.08356)^{2}}{(0.78231)^{2}}}+0.00035 * e^{\frac{x}{0.71944}}+$

$(-2.4398) * \sin ((2.11912) * x)-2.23613$

Now, to check the integrity of our work, we enter this function along with the data of $\mathrm{x}$ section to the Origne software to obtain Y data, and then we draw the graph which is shown in the following:

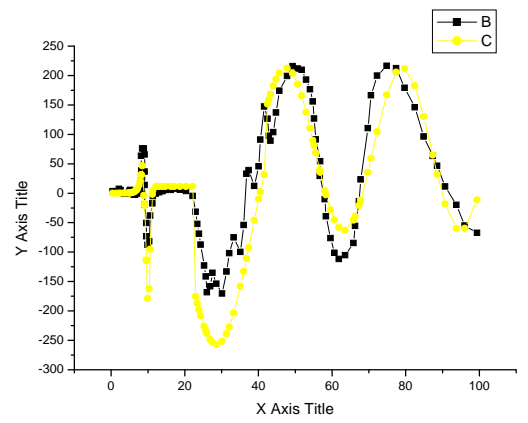

Fig.7. General graph

7. In this figure, two graphs are drawn; the dotted graph plotted by the data of the problem (as in 
figure 2) and the yellow dotted graph is plotted using equations. The two graphs are almost close to each other. V OBTAINING EQUATIONS OF SOUR TASTE SIGNAL

By using recorded signal samples associated with sour taste (Figure 2), we extracted data signals using Getdata software.

This way data are converted to digital form, and we obtain point to point changes.

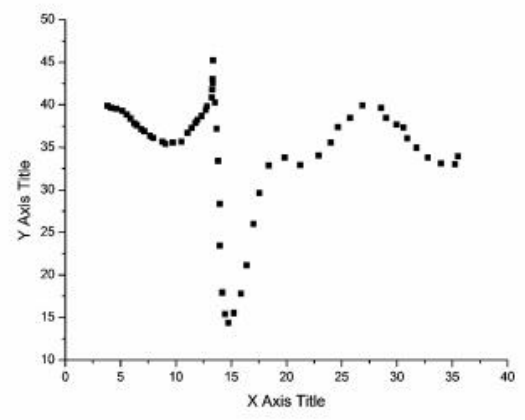

Fig.3. Charting the data after obtaining information

Using the data obtained, we draw a graph of the signal (Fig. 3). We divided the chart into three parts and obtain the function of each section by Origin software. To increase accuracy, we divide the above signal into three intervals and obtain the equation of each section by the software. First interval (4- 14) and for the second interval

Diagram if Section 1, with the following function

$$
Y=54.8815-4.5037 X+0.26446 X^{2}
$$

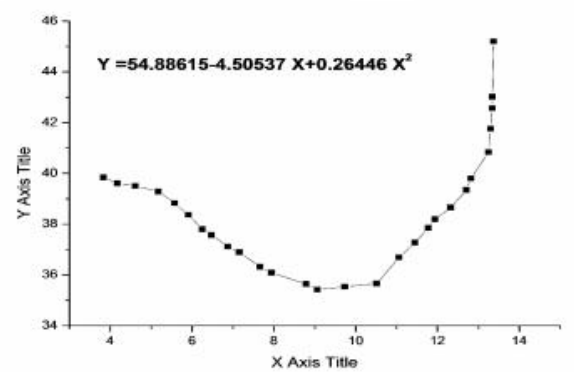

Fig.4. The diagram of the first section in 4-14 range

Diagram Section 2 It's function is a Lorentz Function with the following equation:

$$
y=37.94179+\frac{28(-78.64561)}{\pi} \frac{(1.71188)}{4(x-15.4135)^{2}+(1.71188)^{2}}
$$




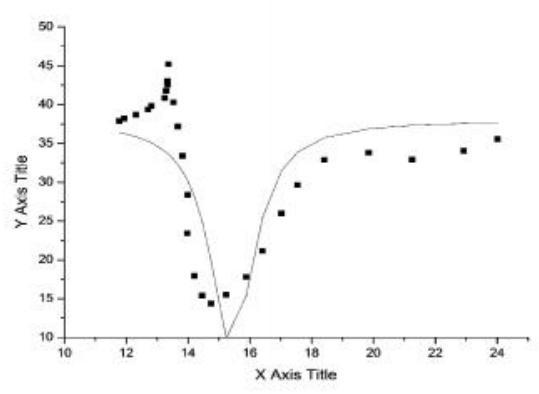

Fig.5. Diagram section 2 in range

Diagram Section 3:

$$
y=33.108424+\frac{44.90684}{5.17451 \sqrt{\frac{\pi}{2}}} e^{\frac{-2(x-27.57322) 2}{5.17451^{2}}}
$$

It has a Gaussian Function with the following equation

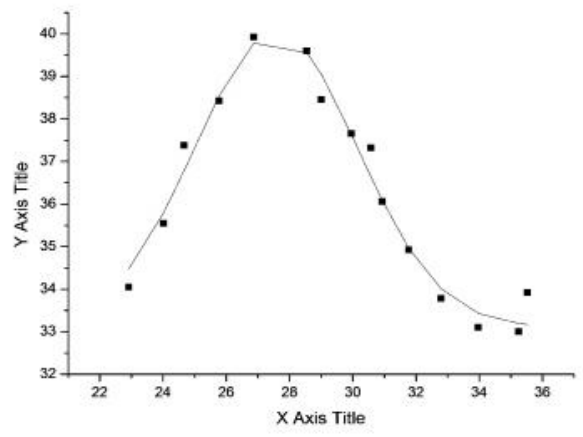

Fig.6. Diagram of section 3 of the range

Finally, the function of the signal can be obtained as follows.

$$
\begin{aligned}
& y=54.88-4.50 x+0.26 x^{2}+37.94 \\
& +\frac{2 *(-78.64)}{\pi} \frac{1.72}{4(x-15.41)^{2}+1.72^{2}}+33.11 \\
& +\frac{44.91}{5.17 \sqrt{\frac{\pi}{2}}} e^{\frac{-2(x-27.57)^{2}}{5.17^{2}}}
\end{aligned}
$$

For comparison, we draw the primary data and obtained equation on a graph as follows: 


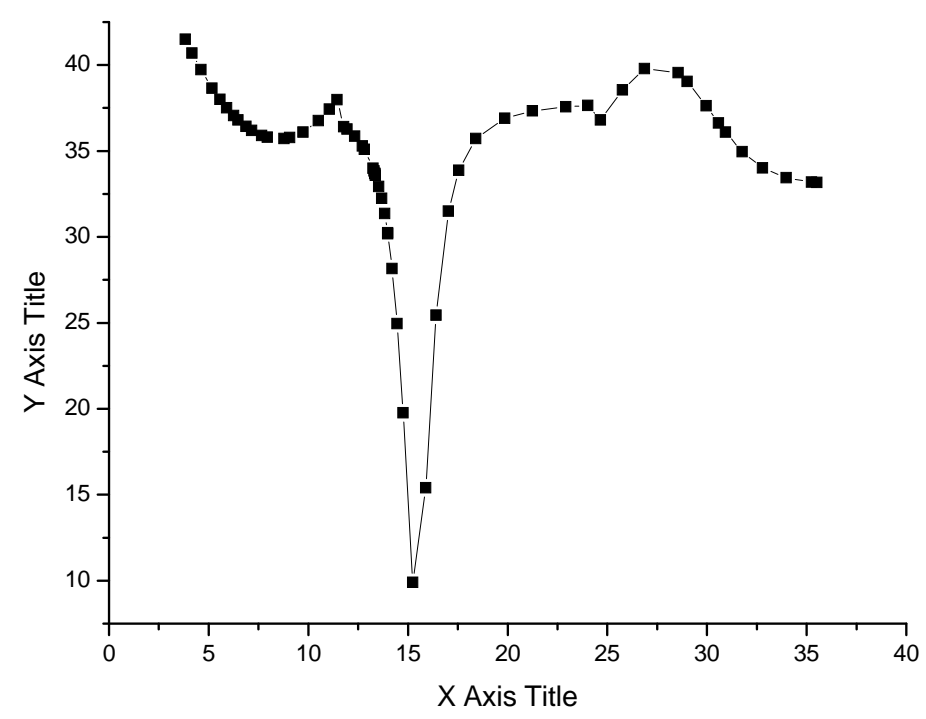

The dotted graph is the initial equation, and the line graph is the obtained graph, as it can be seen, the two graphs are identical

\section{CIRCUIT DESIGN}

According to equation (5) resulted from the signals of the white light (voltage versus time) which were selected from the results of our work, and using Origin software, a program for the above equations with the same intervals was written using Codevision. To increase accuracy, the intervals were selected so that each interval had an overlap with the next interval. By signal optimization, it is possible to design and produce a circuit that is able to simulate the signals of the white light using mathematical methods and software. This electronic unit can produce and simulate potential changes associated with obtained signal equation. The circuit consists of a microcontroller and a voltage indicator. The microcontroller is programmed by Codevision programmer. The circuit can be designed and implemented on an IC, and the IC can be improvised on retinal cells and stimulate retinal cells, so that nerve cells send electrical messages resulted from stimulus to the brain and the brain would receive the signals similar to the white light.

\section{CONCLUSION}

Article reviews signals recorded by experimental work related to retinal cells and shows that we 
can optimize this signal, and create similar potential changes by providing an electronic device. These changes produce identical sense of vision in the nervous system if connected to the nervous area of retinal cells, and this method can be used to make a sense of vision for the blind and visually impaired people.

\section{REFERENCES}

[1] Guyton Ac. Textbook of Medical Physiology. 3rd Ed. Philadelphia: Wb Saunders And Co; 1991.

[2] Holmgren F. Method Att Objectivera Effecten of Ljusintryck $\mathrm{P}^{5}$ Retina, 1865, 1: 184-98.

[3] Dewar J, Mckendrick Jg. On the Physiological Action of Light, Proc. Roy. Soc. (Edinburgh), 1837, 8, 179-82.

[4] Sunness Js. Clinical Retinal Function Testing.

[5] Walsh T.J. Neuro-Ophthalmology: Clinical Signs and Symptoms. 2nd Ed. Philadelphia. Lea and Febiger, 1985, 303-341

\section{How to cite this article:}

Haghighi B, Montazeri M, and Haghighi N. White light signal simulator microcontroller design. J. Fundam. Appl. Sci., 2016, 8(2S), 438-447. 